\title{
Vertebral Fracture Detection Differences of Axial Section Computed Tomography Images and Coronal Sagittal Reformat Images in Trauma Cases
}

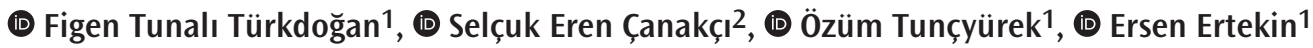 \\ 1Department of Radiology, Aydın Adnan Menderes University Faculty of Medicine, Aydın, Turkey \\ ${ }^{2}$ Clinic of Emergency Medicine, Tavşanlı Doç. Dr. Mustafa Kalemli State Hospital, Kütahya, Turkey
}

\begin{abstract}
Aim: To investigate the contribution of multiplanar sections to vertebral fracture detection by examining the multislice computed tomography (CT) images of patients who were referred to our emergency department (ED) with trauma.

Materials and Methods: We scanned 896 patients who were referred to our ED between 01.06.2016 and 01.06.2018 and whose multislice CTs were taken with a vertebral fracture pre-diagnosis made by the ED physician. The differences between axial and coronal sagittal reformat images in the detection of vertebral fractures were compared in 78 cases that were found with a fracture in their tomographies.

Results: The average age of the 78 cases was $55.93 \pm 23.23$ years. In total, 145 fractures were detected in the 78 cases. Twenty-four fractures were detected in 12 cases in the cervical area, 67 were detected in 36 cases in the thoracic area, and 54 fractures were detected in 30 cases in the lumbar area. While there were no significant differences between axial and coronal sagittal reformat imaging techniques in the differentiation of cervical fractures, differences were found between the techniques for thoracic and lumbar fractures $(p=0.636, p<0.001$, $p=0.001$, respectively). When the fracture detection rates of the axial and coronal sagittal imaging techniques were assessed in terms of the anatomic region, there were significant differences in corpus assessment $(p<0.001)$, while there were no significant differences in transverse process and lamina fractures $(p=0.127, p=0.083)$.

Conclusion: In post-trauma physical examination, coronal sagittal reformat imaging is more sensitive when compared with axial imaging in patients having pain in the thoracic and lumbar region, and we believe that it will useful to physicians in differentiating especially corpus fractures.
\end{abstract}

Keywords: Trauma, coronal sagittal reformat imaging, axial imaging, vertebral fracture

\section{Introduction}

Helical computed tomography (CT) has a significant place in the comparison of bone lesions due to having high resolution. Two and three dimensional reformat images obtained with multislice CT (MSCT), which came out with a two-sliced scanner in 1992 for the first time, found a place in the distinction between muscle and skeletal system (1).

The use of MSCT in trauma, which is a cause of morbidity and mortality in middle and advanced ages, has been increasing gradually $(1,2)$. The purpose of CT use is to be certain of or to exclude a fracture that is suspicious in direct $X$-ray or to guide the treatment by determining the extent of an established fracture. In addition, it shows the bone anatomy in anatomically complex structures such as pelvis, scapula and spine where direct radiography is limited in showing fractures (1).

The combination of three-dimensional (3D) reconstruction formed by using volume rendering (VR) technique and helical CT enables a more detailed analysis of musculoskeletal system and proves to be valuable in the diagnosis and treatment planning of 
a large number of pathologies, especially trauma. This method changes clinical approach in a significant number of cases due to findings that can be assessed only with 3D images or that can be demonstrated better with these images and it is useful in transferring complex spatial information to clinicians correctly and sufficiently (1,3-5). The aim of this study was to investigate the contribution of multiplanar reformat (MPR) sections to the determination of vertebral fracture by reviewing MSCT of the patients presenting with trauma.

\section{Materials and Methods}

Permission was taken from local ethical committee (Adnan Menderes University, 53046469-050,04,04) for this study. Eight hundred and ninety-six cases who referred to the emergency service with trauma between 01.06.2016 and 01.06.2018 and for whom the emergency service physician requested MSCT were scanned. Of these patients, 148 patients whose thoracic CT was performed were included in the study. In all patients, CT scans were performed on a 150-digit Toshiba Acquisition CT device with a 120-KV, automatic mAs selection (115-195 mAs). The field of vision was scanned $2 \mathrm{~cm}$ below the diaphragm from the thorax entrance, completely including the thorax. The section thickness was determined as $3 \mathrm{~mm}$ in axial images and the pitch value was determined as $0.75 \mathrm{~mm}$. From the obtained raw data, sagittal and coronal reformat images of $5 \mathrm{~mm}$ thickness were created. CT images for the fracture scan were evaluated in the bone window $(\mathrm{W} / \mathrm{L}=2500 / 500)$. To avoid bias in the CT assessment, MPR images and axial sections were viewed at different times, unaware of the results, through high-resolution imaging screens. By using the data, vertebral fracture detection differences of axial section CT images and coronal sagittal reformat images were compared.

\section{Results}

Seventy-eight cases who were admitted to the emergency service due to trauma and who were found to have a fracture in their CT were included in the study. Average age of 78 cases was found as $55.93 \pm 23.23$ years. Thirty-six $(46.2 \%)$ of the trauma cases were female. A total of 145 fractures were found in 78 cases. Of these 145 fractures, 24 were detected in 12 cases and in cervical area, 67 were detected in 36 cases and in thoracic area and 54 fractures were detected in 30 cases and in lumbar area (Table 1). When the average ages of the cases were analysed in terms of the area where fracture was found, average age of the cases who were found to have fracture in the cervical area was $45.81 \pm 19.44$ years; average age of the cases who were found to have fracture in the thoracic area was $58.69 \pm 20.62$ years; average age of the cases who were found to have fracture in the lumbar area was $58.23 \pm 24.02$ years and there were no statistical differences between them $(p=0.074)$.

When the areas where fracture was detected and the genders of the cases were examined, two (16.7\%) of the cases with a fracture in the cervical area were female; while $16(44.4 \%)$ of the cases with a fracture in the thoracic area were female and 18 (60.0\%) of the cases with a fracture in the lumbar area were female and significant difference was found only in fractures in cervical and lumbar areas in terms gender $(p=0.006)$.

When the fracture detection rates of axial and coronal sagittal reformat imaging techniques were assessed in terms of fracture area, no significant difference was found between axial and coronal sagittal reformat imaging techniques in terms of the differentiation of cervical fractures $(p=0.636)$; however, the fact that the cases who were detected as no fracture were different shows that both methods can be useful in fracture exclusion in these patient groups (Figure 1 and 2). Significant difference was found between axial and coronal sagittal reformat imaging techniques in the distinction of thoracic and lumbar fractions (Table 2).

When the fracture detection rates of axial and coronal sagittal reformat imaging techniques were assessed in terms of anatomic region, while all of the 117 corpus fractures were seen in coronal sagittal reformat, 58 were seen in axial $(p<0.001)$. When spinous process fractures were assessed, while all of the fractures were seen with both methods, in terms of transverse process fractures, 14 of the 15 fractures were seen in axial, while 11 were seen in coronal sagittal reformat and the difference between was not found to be statistically significant (Table 3).

Table 1. Distribution of fractures and cases according to the region and anatomy of the vertebra

\begin{tabular}{|c|c|c|c|c|}
\hline & & Cervical (12 cases, 24 fractures) & Thoracic (36 cases, 67 fractures) & Lumbar (30 cases, 54 fractures) \\
\hline \multirow{3}{*}{$\begin{array}{l}\text { Anatomic } \\
\text { region }\end{array}$} & Corpus $(n=117)$ & $13(11.1 \%)$ & $60(51.3 \%)$ & $44(37.6 \%)$ \\
\hline & $\begin{array}{l}\text { Spinous process } \\
(n=7)\end{array}$ & $5(71.4 \%)$ & $2(28.6 \%)$ & 0 \\
\hline & $\begin{array}{l}\text { Transverse process } \\
(n=15)\end{array}$ & $1(6.7 \%)$ & $5(33.3 \%)$ & $9(60.0 \%)$ \\
\hline
\end{tabular}




\section{Discussion}

In post-trauma physical examination, coronal sagittal reformat imaging is more sensitive when compared with axial imaging in patients who have tenderness in thoracic and lumbar region and we believe that it will be of use to the physician by distinguishing especially corpus fractures.

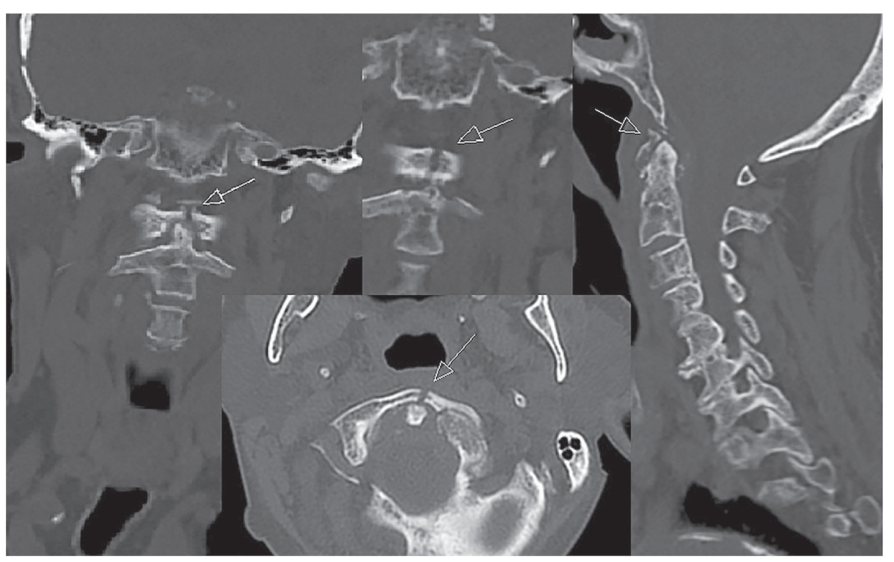

Figure 1. Positive fracture with axial imaging but reformat imaging changes decision

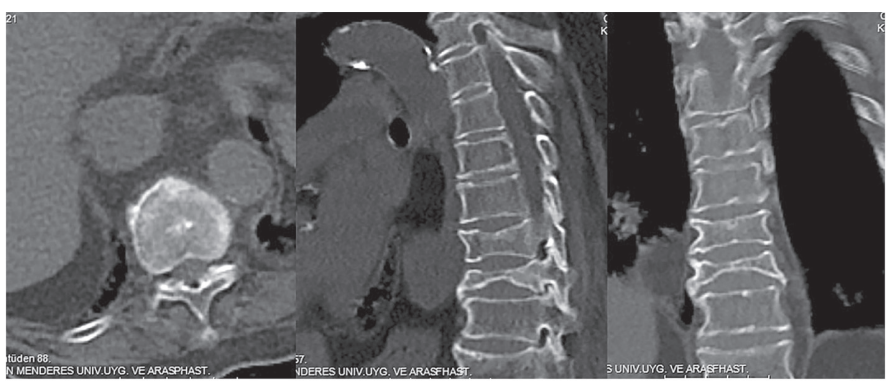

Figure 2. Positive fracture with reformat imaging but axial imaging changes decision
In a study conducted in a centre where MPR and VR 3D images are used routinely in CT examinations in musculoskeletal system trauma, treatment approach was reported to have changed due to findings in MPR or 3D VR images in almost $30 \%$ of patients with pelvic fracture on axial CT images. It has been reported that the reason for this is the presentation of a more severe injury than clinically predicted in MPR or 3D VR images or monitored in conventional axial images (1). In their study, Pate et al. (6) researched the advantages of 3D CT in 202 patients with musculoskeletal system pathology, especially in fractures of the skeletal areas with complex anatomy, articular diseases of the hip and spinal stenosis. They reported that 3D CT does not give additional information to what is obtained with standard radiographic techniques; however, it shows the existing standard data with a different method and the images obtained are more easily understood by clinicians and they are very useful in especially preoperative assessment in most of the cases. In our study, no significant differences were found between axial and coronal sagittal reformat imaging techniques in the differentiation of cervical fractures; however, the fact that the cases who were detected as no fracture were different shows that both methods can be useful in fracture exclusion in these patient groups. Wicky et al. (7) assessed the diagnostic efficacy of direct graphy and 3D helical CT in 42 patients with tibial fracture and the accuracy of these two techniques in planning surgical approach. In the assessment, direct graphy (AP, lateral and both oblique) and 3D images were classified and surgical plan was changed according to the results found. As a conclusion, it was reported that helical $C T$ reconstructions demonstrated tibial plateau fractures better and more correctly and provided more correct surgical planning. However, there are also studies which

Table 2. Fracture detection rates of axial and reformat imaging techniques by fracture areas

\begin{tabular}{|c|c|c|c|c|c|}
\hline & \multicolumn{2}{|c|}{ Axial } & \multicolumn{2}{|c|}{ Coronal sagittal reformat } & p value \\
\hline Cervical $(n=24)$ & $21(87.5 \%)$ & $3(12.5 \%)$ & $22(91.7 \%)$ & $2(8.3 \%)$ & 0.636 \\
\hline Lumbar $(n=54)$ & $39(72.2 \%)$ & $15(27.8 \%)$ & $51(94.4 \%)$ & $3(5.6 \%)$ & 0.001 \\
\hline Total $(n=145)$ & 85 & 60 & 139 & 6 & - \\
\hline
\end{tabular}

Table 3. Fracture detection rates of axial and reformat imaging techniques by anatomic region

\begin{tabular}{|l|l|l|l|l|}
\hline \multicolumn{2}{|c|}{} & Axial & Coronal sagittal reformat & p value \\
\hline \multirow{4}{*}{ Anatomic region } & Corpus $(n=117)$ & $58(68.2 \%)$ & $117(100 \%)$ & $<0.001$ \\
\cline { 2 - 5 } & Spinous process $(n=7)$ & $7(100 \%)$ & $7(100 \%)$ & 0.100 \\
\cline { 2 - 5 } & Transverse process $(n=15)$ & $14(93.3 \%)$ & $11(73.3 \%)$ & 0.127 \\
\cline { 2 - 5 } & Lamina $(n=6)$ & $6(100 \%)$ & $4(66.6 \%)$ & 0.083 \\
\hline n: Number & &
\end{tabular}


show that in tibial plateau fractures, routine CT imaging does not have a contribution to direct graph in classification and treatment plan (8). In their study, Uzun et al. (9) reported that in fractures of the knee region, 3D imaging did not have a contribution to axial CT in terms of the presence of the 3D imaging, articular elongation and the presence of bone fragment; however, 3D VR images were required in the assessment of compression amount in cases which had compression fracture. Deplacement degrees of fragments which can be seen with axial section were assessed better with MPR and 3B VR images.

It has been shown that when assessment in which $2 \mathrm{D}$ imaging and $2 \mathrm{D}$ and $3 \mathrm{D}$ assessment are made together in distal humerus fractures, interobserver compliance increases when 3D evaluation is added (10).

Proximal humerus fracture imaging is primarily conducted with direct graphy. Generally direct graphy is enough in classification and treatment plan conducted by using Neer Classification of two-piece proximal humerus fractures. However, additional information is frequently required in three and four pieces fractures in terms of surgical treatment.

Preoperative CT use with 3D reconstruction has been reported to be valuable in complex fractures (11). In addition, 2D and 3D reconstructions and CT scans are recommended for suspicious wrist traumas where direct radiographic findings are normal (12).

\section{Conclusion}

In conclusion, in the evaluation of trauma patients, coronal sagittal reformat imaging is more sensitive when compared with axial imaging in patients who have tenderness in thoracic and lumbar region and we believe that it will be of use to the physician by distinguishing corpus fractures significantly, especially those which will change the treatment regimen.

\section{Ethics}

Ethics Committee Approval: Permission was taken from local ethical committee (Adnan Menderes University, 53046469$050,04,04)$ for this study.

Informed Consent: Informed consent was obtained from the patients.

Peer-review: Externally peer-reviewed.

\section{Authorship Contributions}

Surgical and Medical Practices: F.T.T., Concept: F.T.T., S.E.Ç., Design: F.T.T., S.E.C.., E.E., Data Collection or Processing: F.T.T., S.E.C.., Ö.T., E.E., Analysis or Interpretation: F.T.T. Ö.T., E.E., Literature Search: F.T.T., S.E.C.., Ö.T., E.E., Writing: F.T.T. Ö.T., E.E.

Conflict of Interest: No conflict of interest was declared by the authors.

Financial Disclosure: The authors declared that this study received no financial support.

\section{References}

1. Pretorius ES, Fishman EK. Volume-rendered three-dimensional spiral CT: musculoskeletal applications. Radiographics. 1999;19:1143-60.

2. Magid D, Fishman EK. Imaging of musculoskeletal trauma in three dimensions. Radiol Clin North Am. 1989;27:945-56.

3. Kuszyk BS, Ney DR, Fishman EK. The current state of the art in threedimensional oncologic imaging: an overview. Int J Radiat Oncol Biol Phys. 1995;33:1029-39.

4. Pretorius ES, Fishman EK. Helical (spiral) CT of the musculoskeletal system. Radiol Clin North Am. 1995;33:949-79.

5. Pretorius ES, Scott WW Jr, Fishman EK. Acute trauma of shoulder: role of spiral CT imaging. Emerg Radiol. 1995;2:13-7.

6. Pate D, Resnick D, Andre M, Sartoris DJ, Kursunoglu S, Bielecki D, et al. Perspective: Three-dimensional imaging of the musculoskeletal system. Am J Roentgenol AJR. 1986;147:545-51.

7. Wicky S, Blaser PF, Blanc CH, Leyvraz PF, Schnyder P, Meuli RA. Comparison between standard radiography and spiral $C T$ with $3 \mathrm{D}$ reconstruction in the evaluation, classification and management of tibial plateau fractures. Eur Radiol. 2000;10:1227-32.

8. te Stroet MAJ, Holla M, Biert J, van Kampen A. The value of a CT scan compared to plain radiographs for the classification and treatment plan in tibial plateau fractures. Emerg Radiol. 2011;18:279-83.

9. Uzun Ç, Atman E, Sahin G. Evaluation of Articular trauma with helical computed tomography: contribution of three-dimensional imaging. I Ankara Univ Fac Med. 2017;70:151-60.

10. Brouwer KM, Lindenhovius AL, Dyer GS, Zurakowski D, Mudgal CS, Ring D. Diagnostic accuracy of 2- and 3-dimensional imaging and modeling of distal humerus fractures. J Shoulder Elbow Surg. 2012;21:772-6.

11. Bahrs C, Rolauffs B, Südkamp NP, Schmal H, Eingartner C, Dietz K, Pereira PL, Weise K, Lingenfelter E, Helwig P. Indications for computed tomography (CT-) diagnostics in proximal humeral fractures: a comparative study of plain radiography and computed tomography. BMC Musculoskelet Disord. 2009;10:33.

12. You JS, Chung SP, Chung HS, Park IC, Lee HS, Kim SH. The usefulness of CT for patients with carpal bone fractures in the emergency department. Emerg Med J. 2007;24:248-50 